\title{
Immune checkpoints: Cytotoxic T-lymphocyte antigen 4 and programmed cell death protein 1 in breast cancer surgery
}

\author{
AGNIESZKA KOLACINSKA ${ }^{1,2^{*}}$, BARBARA CEBULA-OBRZUT $^{3 *}$, LUKASZ PAKULA $^{4 *}$, \\ JUSTYNA CHALUBINSKA-FENDLER ${ }^{5}$, ALINA MORAWIEC-SZTANDERA ${ }^{1}$, ZOFIA PAWLOWSKA ${ }^{6}$, \\ IZABELA ZAWLIK $^{7,8}$, ZBIGNIEW MORAWIEC ${ }^{2}$, DOROTA JESIONEK-KUPNICKA ${ }^{9}$ and PIOTR SMOLEWSKI ${ }^{3}$ \\ ${ }^{1}$ Department of Head and Neck Cancer Surgery, Medical University of Łódź; ${ }^{2}$ Department of Surgical Oncology, \\ Cancer Center, Łódź 93-509; ${ }^{3}$ Department of Experimental Hematology, Medical University of Łódź, Łódź 93-510; \\ ${ }^{4}$ Department of Anesthesiology, Copernicus Memorial Hospital; ${ }^{5}$ Department of Radiotherapy, Medical University of Łódź, \\ Łódź 93-509; ${ }^{6}$ Central Scientific Laboratory, Medical University of Łódź, Łódź 92-215; ${ }^{7}$ Department of \\ Medical Genetics, Institute of Nursing and Health Sciences, Faculty of Medicine, University of Rzeszów; \\ ${ }^{8}$ Center for Innovative Research in Medical and Natural Sciences, Rzeszów 35-959; ${ }^{9}$ Department of \\ Cancer Pathology, Medical University of Łódź, Łódź 93-509, Poland
}

Received August 8,2014; Accepted April 9, 2015

DOI: $10.3892 / \mathrm{ol} .2015 .3321$

\begin{abstract}
Immune checkpoints refer to a plethora of inhibitory pathways built into the immune system, and recent studies have emphasized the role of these checkpoints in carcinogenesis. The aim of the present study was to evaluate two major immune checkpoints, the cytotoxic T-lymphocyte antigen 4 (CTLA-4) and programmed cell death protein 1 (PD-1), in the serum of 35 patients with stage I and II breast cancer. Serum concentrations of CTLA-4 and PD-1 were measured at three time points: i) Preoperatively; ii) during anesthesia following the harvesting of sentinel nodes (SNs); and iii) $24 \mathrm{~h}$ postoperatively. Control samples were obtained from 25 healthy, age-matched females. Assessment of CTLA-4 and PD-1 expression levels was conducted using flow cytometry. A statistically significant difference in PD-1 expression was identified between breast cancer patients preoperatively and healthy controls $(26.31 \pm 11.87$ vs. $12.72 \pm 8.15 ; \mathrm{P}<0.0001)$. In addition, a statistically significant association was found between CTLA-4 and PD-1 levels prior to surgery $(\mathrm{P}=0.0084)$. In addition, CTLA-4 expression was associated with age $(\mathrm{P}=0.0453)$, with elevated levels of CTLA-4 detected in older breast cancer patients. Higher PD-1 expression levels were observed in T2 tumors compared with T1 tumors prior to surgery and intraoperatively; however, the differences were not statistically significant. Furthermore, a
\end{abstract}

Correspondence to: Professor Agnieszka Kolacinska, Department of Head and Neck Cancer Surgery, Medical University of Łódź, 4 Paderewskiego, Łódź 93-509, Poland

E-mail: agnieszka.kolacinska@umed.lodz.pl

*Contributed equally

Key words: breast cancer, cytotoxic T-lymphocyte antigen 4, programmed cell death protein 1 decrease in PD-1 levels was observed subsequent to harvesting SNs with metastasis, but not in $\mathrm{SN}$-negative patients $(\mathrm{P}=0.05)$. A negative correlation was also observed between PD-1 expression and progesterone receptor (PR) status following surgery $(\mathrm{P}=0.024)$. These results provided a basis for further investigation of immune checkpoints in breast cancer. Breast cancer patients exhibit an altered profile of immune checkpoint markers, with higher concentrations of PD-1 observed in larger, PR-negative tumors.

\section{Introduction}

The value of lymph node surgery in breast cancer patients has been greatly debated over the past years, resulting in a wide range of management techniques, including radical excision of three levels of axillary lymph nodes and parasternal nodes (1), axillary lymph node dissection (2), sentinel node biopsy (3), omission of axillary lymph node dissection in cases of macroor micrometastases in selected cases $(4,5)$, and abandonment of nodal procedures in early-stage breast cancer $(6,7)$. Despite a paradigm shift and progressive decline in the extent of lymph node surgery in recent years, the survival rates in breast cancer patients have improved and axillary recurrence remains extremely low $(\sim 1 \%$ per 5 years) $(4,5)$. The improvement in survival rates may be due to following: i) Modern postoperative adjuvant therapies, including chemo-, endocrine, anti-human epidermal growth factor receptor 2 (HER2) and radio-therapy, may eliminate low volume axillary metastases in residual nodes in early-stage breast cancer; ii) intact axillary lymph nodes may eliminate low-volume disease by immune surveillance mechanisms in early-stage breast cancer; or iii) presence of stem cells may be required in the sentinel lymph for regional or systemic relapse to occur (4-6). The aims of the present study are based on the second hypothesis, with an emphasis on how the removal of sentinel nodes (SN), with and without metastasis, may influence immune checkpoints. 
Immune checkpoints refer to a plethora of inhibitory pathways built into the immune system, which are crucial for the maintenance of self-tolerance and modulation of the duration and amplitude of physiological immune responses in peripheral tissue, in order to minimize collateral tissue damage $(8,9)$. The blockade of immune checkpoints using monoclonal antibodies directed at the inhibitory immune receptors, including cytotoxic T-lymphocyte antigen 4 (CTLA-4) and programmed cell death protein 1 (PD-1), has emerged as a successful treatment approach for patients with advanced melanoma, lung cancer and kidney cancer [for instance, ipilimumab (anti-CTLA-4), and nivolumab or lambrolizumab (anti-PD1)] (10-12).

CTLA-4 (also known as CD152) is a surface protein of $\mathrm{T}$ cells that downregulates the immune system, leading to inhibition of T cell activation. CTLA-4 stimulation functions as a 'switch-off' for T cell attack on the antigen at the time of the initial response phase, primarily by naïve cells (8). CTLA-4 blockade is important for the inhibition of immune tolerance against tumor cells. One of the hallmarks of CTLA-4 blockade is the durability of objective tumor response (8).

PD-1 (also known as CD279) is a membrane protein expressed on the surface of activated T cells, B cells, natural killer cells and macrophages, negatively regulating the immune response. Whilst CTLA-4 is operational during the early activation of T naïve cells in lymphatic tissues, PD-1 functions during the effector phase of $\mathrm{T}$ cell activation. The interaction of PD-1 with its ligands occurs predominantly in peripheral tissues, such as in the tumor microenvironment, resulting in apoptosis and downregulation of the experienced $\mathrm{T}$ cell effector function (8).

The concept of immune checkpoints in breast cancer is a growing field of interest. During the San Antonio Breast Cancer Symposium in December 2013 (www.sabcs.org), and the European Breast Cancer Conference in March 2014 (www. ecco-org.eu), Loi et al (13) concluded that higher levels of the immune negative regulators, CTLA-4 and PD-1, enhanced the benefit of trastuzumab therapy in HER2-positive breast cancer patients. Other immune modulators, including adenosine and adenosine receptors, in combination with chemotherapy are currently investigated in aggressive triple negative subtypes (14). Furthermore, Denkert et al (15) (American Society of Medical Oncology meeting, May/June 2014; www.asco.org) reported that the expression of selected immune markers [including CTLA-4, PD-1 and PD-ligand 1 (PD-L1)], in addition to tumor infiltrating lymphocytes, may be used to identify patients who exhibit an enhanced response to neoadjuvant carboplatin/doxorubicine/taxane chemotherapy in HER-positive and triple negative breast cancer patients. Accumulating evidence revealed that a combination of CTLA-4 or PD-1/PD-L1 blockade targeted jointly with radiotherapy significantly reduced the tumor size and improved the overall survival $(16,17)$. Other studies have reported that immune checkpoint blockade and endocrine therapy may have a synergistic effect in the treatment of various types of cancer (18). However, only a limited number of studies exist on the precise role and effect of immune checkpoints in breast cancer surgery, particularly axillary lymph node surgery.

The aim of the current study was to evaluate selected immune checkpoints (CTLA-4 and PD-1) preoperatively in the serum of breast cancer patients in comparison with healthy controls. In addition, the association of CTLA-4 and PD-1 perioperatively in the serum of breast cancer patients with various clinicopathological factors, including age, tumor size, receptor status [estrogen receptor (ER), progesterone receptor (PR) and HER2] and SN status, was determined.

\section{Patients and methods}

\section{Patients}

Inclusion criteria. A total of 35 female patients with operable stage I-II breast cancer, suitable for breast-conserving surgery (BCS) and SN biopsy (SNB), were enrolled in the study. The patients had undergone surgery in the Department of Surgical Oncology, Cancer Center (Łódź, Poland) between September and December 2013. Blood samples $(2.7 \mathrm{ml})$ were collected to determine the levels of CTLA-4 and PD-1 at three time points: i) Preoperatively; ii) during anesthesia following the harvesting of SNs; and iii) $24 \mathrm{~h}$ postoperatively. Control blood samples were obtained from 25 healthy, age-matched females. All the patients provided written informed consent, and approval was obtained from the Ethics Committee of the Institutional Review Board of the Medical University of Łódź, Poland (No. RNN/239/13/KE).

Exclusion criteria. Patients with concomitant or previous autoimmune diseases, other immune disorders or a medical history of other malignancies were excluded from the study. Pregnant or lactating females were also ineligible.

CTLA-4 and PD-1 assessment. CTLA-4 and PD-1 expression was assessed using flow cytometry (FACSCanto ${ }^{\mathrm{TM}}$ II; BD Biosciences, San Diego, CA, USA). The following reagents (all purchased from BD Biosciences) were used: Phycoerythrin (PE)-labeled monoclonal mouse anti-human CD152 (CTLA-4) antibody (clone BNI3; catalog no. 555853); PE-labeled mouse $\mathrm{IgG}_{2 \mathrm{a}}, \kappa$ isotype control antibody (catalog no. 555574); Cytofix/Cytoperm ${ }^{\mathrm{TM}}$ Fixation/Permabilization Solution kit; PE-labeled monoclonal mouse anti-human CD279 (PD-1) antibody (clone EH12.1; catalog no. 560795); PE-labeled mouse $\operatorname{IgG}_{1} \kappa$ isotype control antibody (catalog no. 554680); and Pharm Lyse ${ }^{\mathrm{TM}}$ lysis buffer. All the procedures were conducted following the manufacturer's instructions. The cells were incubated with surface monoclonal mouse anti-human CD3 (catalog no. 345763), CD8 (catalog no. 345774), CD279 (PD-1; catalog no. 560795) and CD152 (CTLA-4; catalog no. 555853) antibodies conjugated with the fluorochromes allophycocyanin (APC), peridinin chlorophyll protein (Per-CP), and PE, respectively, (all purchased from BD Biosciences) at a concentration of $20 \mu 1 / 10^{6}$ cells, in the dark at room temperature for $15 \mathrm{~min}$. Next, $1 \mathrm{ml}$ lysis buffer was added, incubated in the dark at room temperature for $15 \mathrm{~min}$, and washed in phosphate-buffered saline (PBS; PAA Laboratories GmbH, Pasching, Austria). The cells were subsequently fixed and permeabilized using an intracellular staining kit according to the manufacturer's protocol (BD Biosciences). The cells were then incubated with monoclonal mouse anti-human PE-conjugated antibodies against intracellular CD152 (catalog no. 555853; BD Biosciences), and their corresponding isotype controls, in the dark at room temperature for $30 \mathrm{~min}$. Subsequently, the samples were washed in PBS and assessed using a FACSCanto ${ }^{\mathrm{TM}}$ II flow cytometer. 

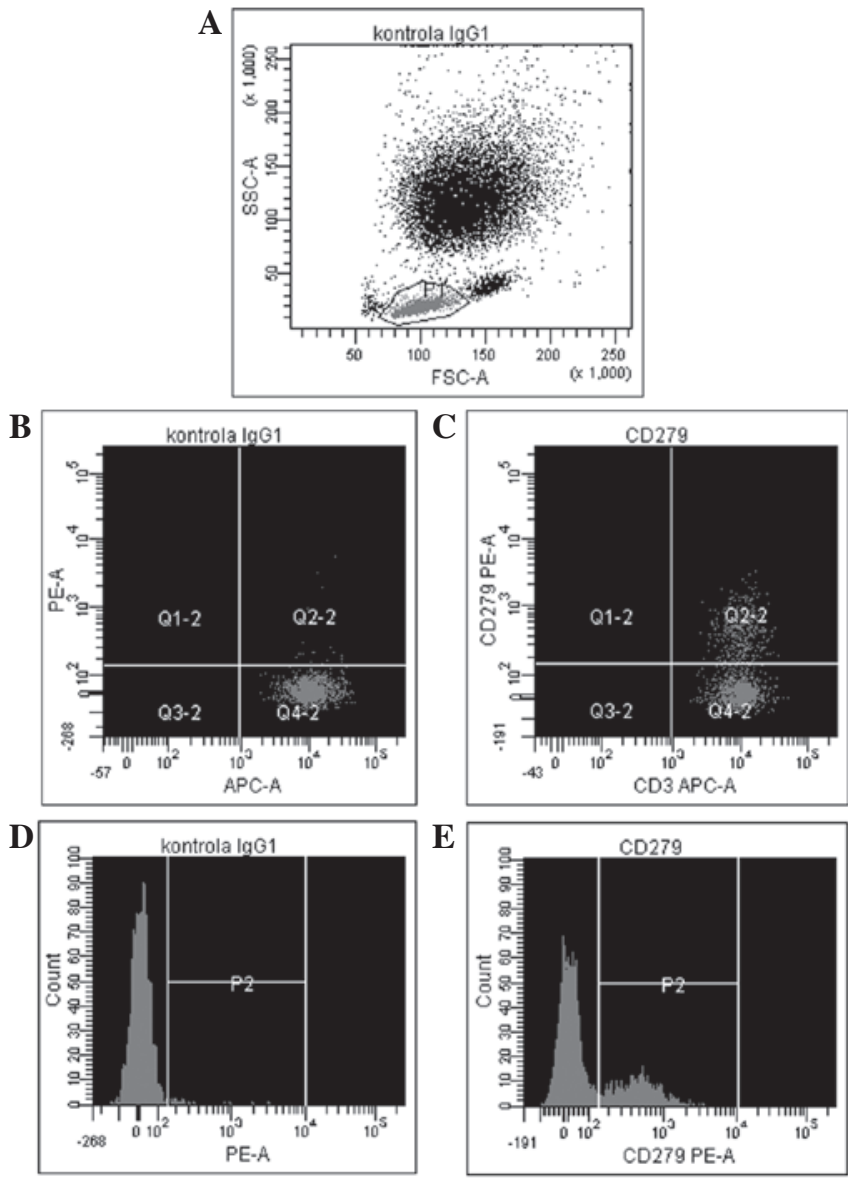

Figure 1. A representative example of PD-1 assessment using flow cytometry. (A) the lymphocyte population was gated using forward scatter vs. side scatter. (B) isotype control for PD-1 (CD279) antigen measured on CD3+ cells, and (C) expression of PD-1 on CD3+ lymphocytes from the patient sample. Representative histograms showing (D) isotype control for PD-1 antigen and (E) PD-1 expression in the patient sample. CD279 (PD-1), programmed cell death protein 1; kontrola, control; APC-A, allophycocyanin PE-A, phycoerythrin; FSC-A, forward scatter area; SSC-A, side-scatter area; IgG, immunoglobulin.

Pathological assessment. Postoperative specimens were subjected to a routine examination of tumor size (T stage), type, and grade according to the Elston-Ellis modification of the Bloom-Richardson scale (19), and sentinel lymph node status.

HER2 and ER/PR status was evaluated by immunohistochemistry (IHC) or by fluorescence in situ hybridization (FISH). Briefly, the breast cancer tissue samples were fixed in $10 \%$ neutral buffered formalin, paraffin-embedded and cut into $4 \mu \mathrm{m}$ sections, then stained with hematoxylin and eosin. IHC for ER and PR was performed using the Envision System (Dako North America, Inc., Carpinteria, CA, USA) and Dako Auto-Stainer Plus (Dako North America, Inc.). Staining was assessed according to Allred method (20) in which two features were assessed; The proportion of positive cells (IP) and the intensity of staining (IS). The IP was scored as follows: 0 , no positive cells; $1, \leq 1 \%$ positive cells; $2, \leq 10 \%$ positive cells; $3, \leq 33 \%$ positive cells; $4, \leq 66 \%$ positive cells; and $5,>66 \%$ positive cells. The IS was assessed on a scale from 0 (no staining) to 3 (strong reaction). A total score (TS) for staining was then determined by calculating the sum of the IP and IS scores. Samples exhibiting a TS of 0-2 were regarded
Table I. Breast cancer patient characteristics $(n=35)$.

\begin{tabular}{lr}
\hline Characteristic & Value \\
\hline Age, years & \\
Median & 61.2 \\
Range & $28-8$ \\
Tumor type, $\mathrm{n}$ & \\
No special type & 30 \\
Lobular & 4 \\
Mucinous & 1 \\
Tumor grade ${ }^{\mathrm{a}}, \mathrm{n}$ & \\
G2 & 19 \\
G3 & 12
\end{tabular}

Pathological tumor size, $n$ pT1

pT2 $(>2 \mathrm{~cm}, \leq 5 \mathrm{~cm})$

Tumor size, $\mathrm{cm}$

Median

Range

Number of sentinel nodes removed

Median

Range

Sentinel node status, $\mathrm{n}$

pNO

pNmic

pN1

3

$\mathrm{pN} 2$

1

Receptor status, $\mathrm{n}$

ER-positive

ER-negative

PR-positive

PR-negative

HER2-positive

HER2-negative

HER2-unknown

${ }^{a}$ Elston-Ellis modification of Bloom-Richardson scale. pT1, tumors $\leq 2 \mathrm{~cm}$; pT2, tumors $>2 \mathrm{~cm}$ and $\leq 5 \mathrm{~cm}$; pN0, lymph node without metastases; pN1mic, lymph node with micrometastasis $(>0.2 \mathrm{~mm}$, $\leq 2 \mathrm{~mm})$; pN1, macrometastases $(>2 \mathrm{~mm}$ ) in 1-3 lymph nodes; $\mathrm{pN} 2$, macrometastases in 4-9 lymph nodes; ER, estrogen receptor; PR, progesterone receptor; HER2, human epidermal growth factor receptor 2 .

as negative, while samples exhibiting scores between 3 and 8 were considered as positive.

For HER-2 IHC, HER-2 protein expression was detected using a iVIEW DAB Detection kit (Ventana Medical Systems, Inc., Tucson, AZ, USA) and a BenchMark BX automated slide staining instrument (Ventana Medical Systems, Inc.). HER-2/neu membrane staining was evaluated according to the manufacturer's instructions by a qualified pathologist in accordance with the recommendations for HER2 Testing in Breast Cancer: ASCO/CAP Guideline Update (21). Staining 
Table II. PD-1 levels preoperatively in breast cancer patients and healthy controls. Higher levels were found in breast cancer patients $(\mathrm{P}<0.0001)$.

PD-1 expression, \% positive cells

\begin{tabular}{lccccrrr}
\cline { 3 - 6 } Group & $\mathrm{n}$ & Mean & $\mathrm{SD}$ & Median & $\mathrm{Q}_{1}$ & $\mathrm{Q}_{3}$ & P-value \\
\hline Cancer & 35 & 26.31 & 11.87 & 24.80 & 17.20 & 34.60 & $<0.0001$ \\
Control & 25 & 12.72 & 8.15 & 11.00 & 6.50 & 14.90 &
\end{tabular}

PD-1, programmed cell death protein 1; SD, standard deviation; $\mathrm{Q}_{1}$, first quartile; $\mathrm{Q}_{3}$, third quartile.

was scored as follows: 0, negative (no membrane staining); $1+$, negative (faint, partial staining of the membrane in any proportion of the cancer cells); $2+$, equivocal (weak to moderate complete staining of the membrane in $>10 \%$ of cancer cells); and $3+$, positive (strong, complete staining of the membrane in $>30 \%$ of cancer cells).

Subsequently, FISH was performed on tissue sections with an IHC score of 2+ for HER-2. HER-2 gene status was determined using the PathVysion ${ }^{\circledR}$ HER-2 DNA Probe and Paraffin Pretreatment kits (Abbott Laboratories, Abbott Park, IL, USA) according to the manufacturer's instructions. Fluoresence was detected using a fluorescence microscope (BX51; Olympus Corporation, Tokyo, Japan). For all tumor specimens, the HER 2 and centromere 17 (CEN-17) signals from 20 nuclei were counted and the HER2/CEN-17 ratios were calculated. The samples were considered positive for gene amplification when the HER2/CEN17 ratio was $>2.0$ or the average number of HER2 signals per cell was $>6.0$. Conversely, the samples were considered negative for gene amplification if the HER2/ CEN-17 ratio was $<1.8$ or the average number of HER 2 signals per cell was $<4.0$. In cases exhibiting intermediate results (HER2/CEN-17 ratio, 1.8-2.0) with an average number of HER2 signals per cell of $>6.0$, an additional 20 cells were counted. When the results were in the same range the samples were defined as unequivocal.

Samples were considered ER/PR negative if $<1 \%$ of the tumor cells were immunoreactive. Samples were considered HER2 negative with IHC 1+ staining or with a score of 2+ and no HER2 gene amplification when assessed by FISH.

Statistical analysis. Data are presented as the median with 25-75 percentile $\left(Q_{1}-Q_{3}\right)$ boundaries, unless otherwise stated. Mann-Whitney's U test was used for pairwise comparisons. Spearman's rank correlation coefficient was used to evaluate associations between continuous variables. Non-parametric analysis of variance for repeated measures (Friedman's test) was used for the comparison of several time-points. Post-hoc tests were performed with the Bonferroni-adjusted Wilcoxon's signed rank test. $\mathrm{P}<0.05$ was considered to indicate a statistically significant difference. Statistica 10 software (StatSoft Inc., Tulsa, OK, USA) was used for statistical analysis.

\section{Results}

Patient characteristics. Detailed patient characteristics are listed in Table I, including age, tumor size, receptor status and SN status.

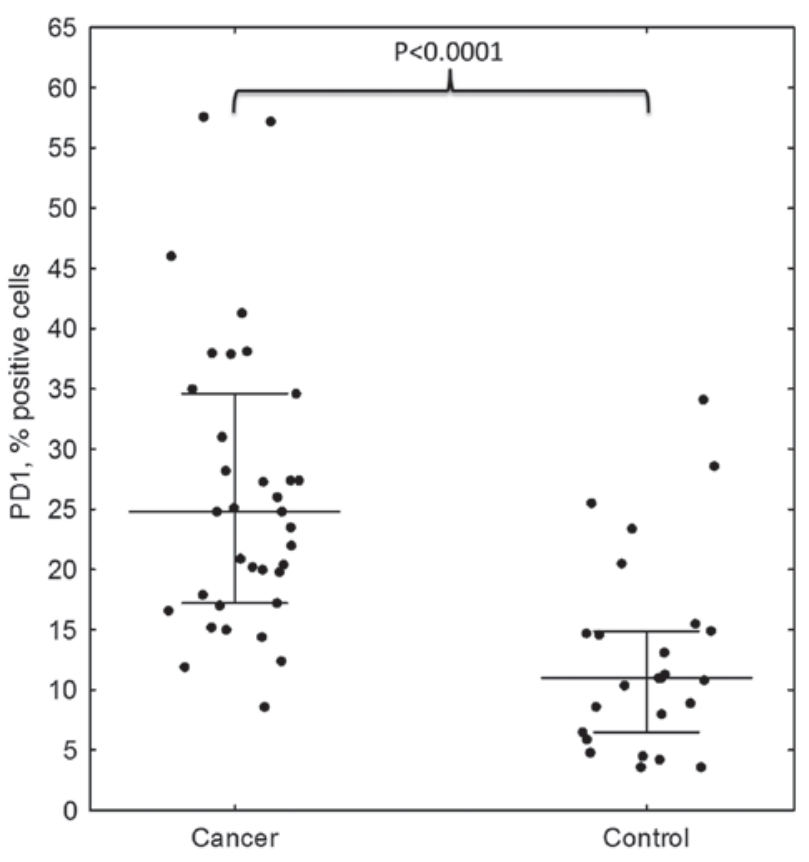

Figure 2. PD-1 expression in breast cancer patients and healthy controls assessed by flow cytomtery. Higher PD-1 levels were found in breast cancer patients compared with controls $(\mathrm{P}<0.0001)$. PD-1, programmed cell death protein 1 .

CTLA-4 and PD-1 expression levels. The results of the flow cytometric analysis of PD-1 (CD279) expression for a representative sample are shown in Fig. 1. A statistically significant difference in PD-1 expression was identified between preoperative breast cancer patients and healthy controls (mean \pm standard deviation, $26.31 \pm 11.87 \%$ vs. $12.72 \pm 8.15 \%$, respectively; $\mathrm{P}<0.0001$; Fig. 2, Table II). In breast cancer patients, the expression levels of PD-1 differed significantly between the various time-points ( $\mathrm{P}=0.0458)$, with the difference between the second and third point being the most significant $(\mathrm{P}=0.0007)$. The median PD-1 levels were $24.80 \%\left(\mathrm{Q}_{1}-\mathrm{Q}_{3}, 17.20-34.60 \%\right)$ prior to surgery and $24.65 \%\left(\mathrm{Q}_{1}-\mathrm{Q}_{3}, 19.50-36.85 \%\right)$ during surgery, decreasing to $21.25 \%\left(\mathrm{Q}_{1}-\mathrm{Q}_{3}, 17.75-32.60 \%\right) 24 \mathrm{~h}$ after surgery. No statistically significant differences were noted in CTLA-4 expression, which remained constant over the time points assessed $(\mathrm{P}=0.3788)$. In addition, no correlation was identified in CTLA-4 expression between the breast cancer patients and healthy individuals. By contrast, a statistically significant association was observed between CTLA-4 and PD-1 levels prior to surgery in breast cancer patients $(\mathrm{r}=0.43 ; \mathrm{P}=0.0084)$; however, 


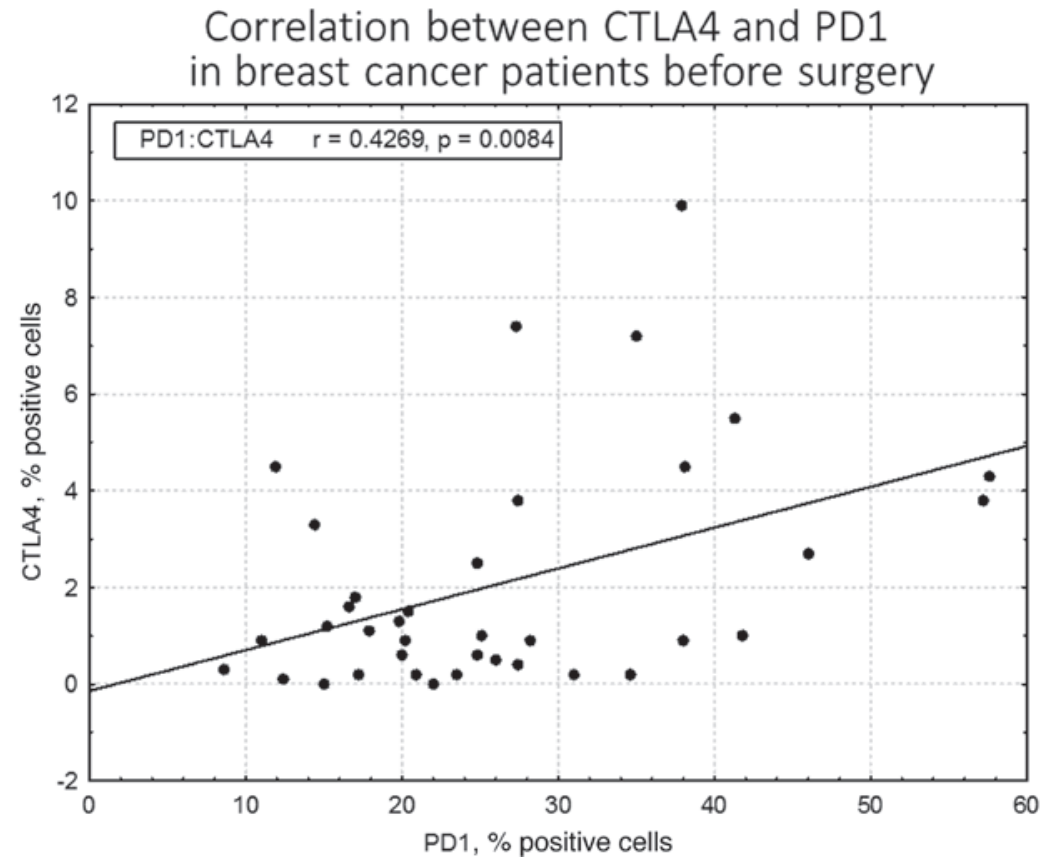

Figure 3. Correlation between CTLA-4 and PD-1 expressions in breast cancer patients prior to surgery, assessed by flow cytometry. A statistically significant correlation was identified between CTLA-4 and PD-1 levels prior to surgery ( $r=0.43$; $\mathrm{P}=0.0084)$. CTLA-4, cytotoxic T-lymphocyte antigen 4; PD-1, programmed cell death protein 1 .

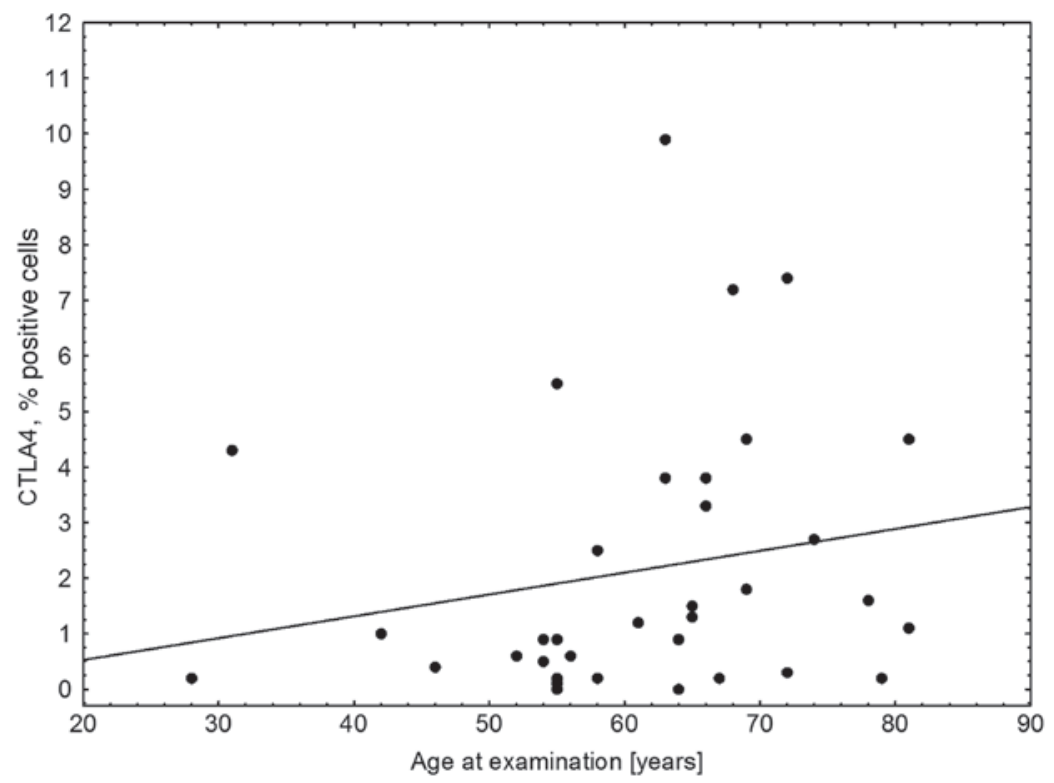

Figure 4. Correlation between CTLA-4 expression, as assessed by flow cytometry, and age in breast cancer patients. Elevated levels of CTLA-4 were present in older breast cancer patients $(\mathrm{r}=0.33 ; \mathrm{P}=0.0453)$. CTLA-4, cytotoxic T-lymphocyte antigen 4.

this disappeared in the two subsequent measurements, during $(\mathrm{r}=0.08 ; \mathrm{P}=0.62)$ and after surgery $(\mathrm{r}=0.14 ; \mathrm{P}=0.43$; Fig. 3$)$. CTLA-4 expression was associated with age $(r=0.33 ; \mathrm{P}=0.0453)$, with elevated levels of CTLA-4 expression observed in older breast cancer patients (Fig. 4). The association of PD-1 levels and tumor size was also analyzed, with higher levels observed in $\mathrm{T} 2$ tumors compared with $\mathrm{T} 1$ tumors prior to surgery (T2 vs. T1, $31.41 \pm 14.14 \%$ vs. $22.47 \pm 8.28 \% ; \mathrm{P}=0.07)$ and intraoperatively ( $\mathrm{T} 2$ vs. $\mathrm{T} 1,32.81 \pm 13.21 \%$ vs. $24.61 \pm 10.68 \%$; $\mathrm{P}=0.08$; however, the differences were not statistically significant (Fig. 5). Furthermore, a decrease in PD-1 levels was observed subse- quent to harvesting SNs with metastasis, but not in SN-negative patients ( $\mathrm{P}=0.05$; Fig. 6). A negative correlation between $\mathrm{PD}-1$ expression and PR status was detected following BCS and SNB $(\mathrm{r}=-0.39 ; \mathrm{P}=0.024)$.

\section{Discussion}

In the current study, a striking difference $(\mathrm{P}<0.0001)$ was observed in immune checkpoint PD-1 expression between breast cancer patients and healthy controls, with significantly lower levels in the latter group. This may be expected as the 


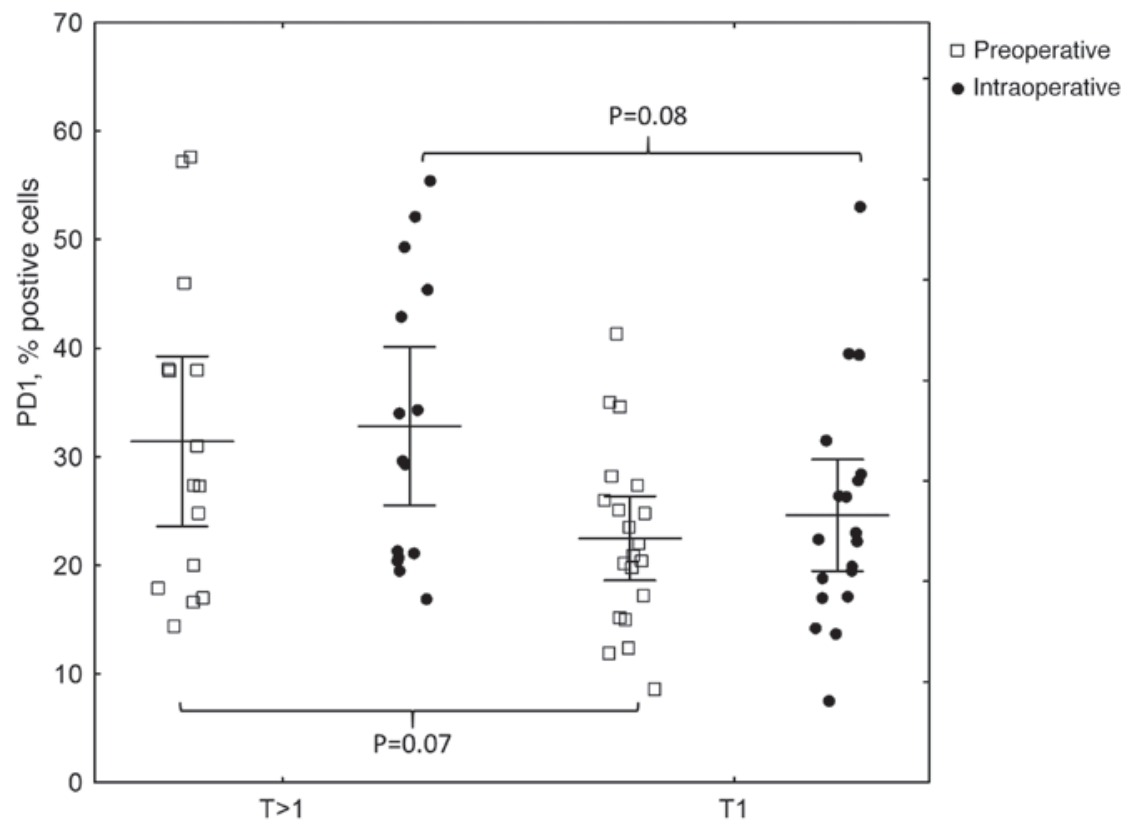

Figure 5. Correlation between PD-1 expression (as assessed by flow cytometry) and tumor size. Higher PD-1 levels were observed in T2 tumors compared with $\mathrm{T} 1$ tumors prior to surgery (T2 vs. $\mathrm{T} 1,31.41 \pm 14.14$ vs. $22.47 \pm 8.28 ; \mathrm{P}=0.07$ ) and intraoperatively (T2 vs. $\mathrm{T} 1,32.81 \pm 13.21$ vs. $24.61 \pm 10.68 ; \mathrm{P}=0.08$ ); however, the differences were not statistically significant. PD-1, programmed cell death protein 1.

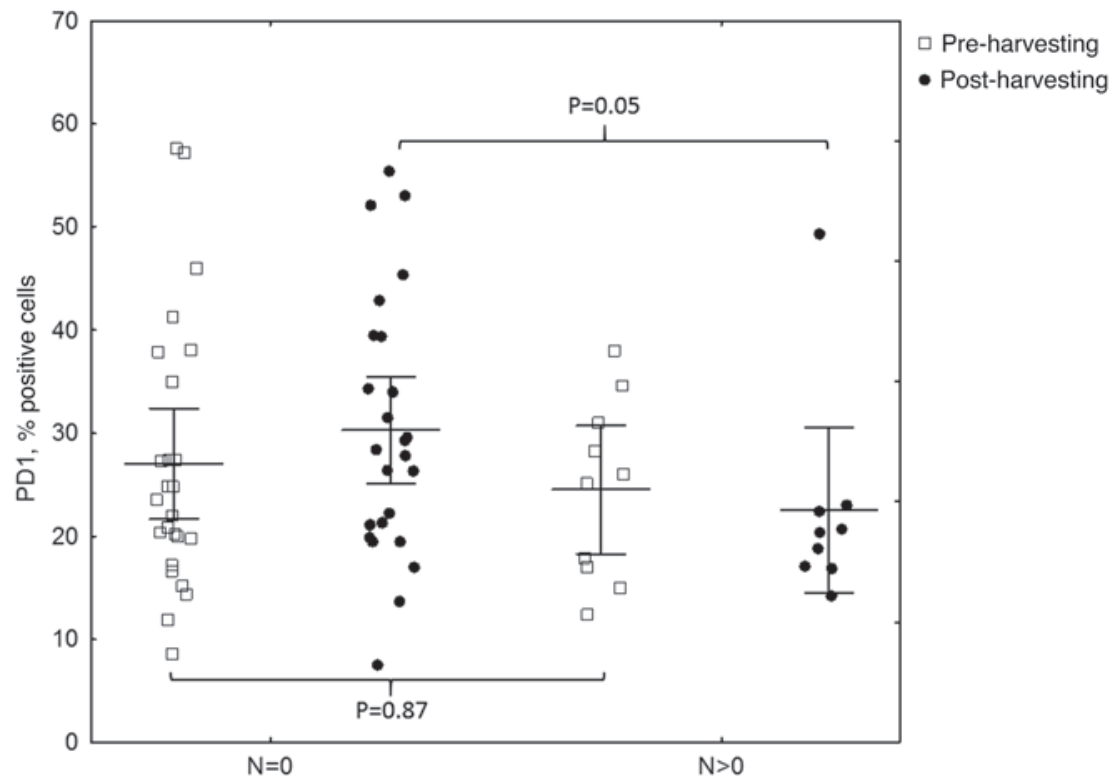

Figure 6. Correlation between PD-1 expression and SN status [with $(\mathrm{N}>0)$ vs. without $(\mathrm{N}=0)$ metastasis]. A decrease in PD-1 levels was observed subsequent to harvesting SN with metastasis, but not in SN-negative patients ( $\mathrm{P}=0.05)$. PD-1, programmed cell death protein 1; SN, sentinel node.

immune system is often impaired in cancer patients, and inhibitory immune pathways are predominant. This is consistent with the observations of Legat et al (22), that expression of inhibitory co-receptors, including PD-1, CTLA-4, LAG3 and CD160, is generally considered a hallmark of T cell exhaustion, particularly in the context of chronic antigen exposure, such as persistent viral infection or cancer. Poschke et al (23) noted tumor-induced changes in the phenotype of blood-derived and tumor-associated $\mathrm{T}$ cells in 43 patients with stage I and II breast cancer, compared with 10 patients with benign disease. These findings were most pronounced in $\mathrm{CD} 8^{+} \mathrm{T}$ cells, which are key components of tumor immune surveillance. Blood from early-stage breast cancer patients contained fewer naïve and more antigen-experienced memory $\mathrm{T}$ cells compared with healthy controls. The aforementioned observations are consistent with those of the current study, which were also performed in stage I and II breast cancer patients, with isolation of $\mathrm{CD}^{+}$ $\mathrm{T}$ cells and PD-1 expression associated primarily with experienced $\mathrm{T}$ cells. In more advanced disease (stages III and IV), higher tumor burden and metastatic spread induce further inhibition and dysfunction of immune surveillance (23). Azim et al (24) demonstrated that aggressive pregnancy-associated breast cancer patients exhibited higher expression levels of inhibitory PD-1 $(\mathrm{P}=0.015)$ and its ligand PD-1L $(\mathrm{P}=0.014)$ 
compared with non-pregnant breast cancer patients. In the present study, patients with pregnancy-associated breast cancer were not included.

The association between immune checkpoint expression and age of breast cancer patients was also examined. CTLA-4 expression was found to be associated with age $(r=0.33$; $\mathrm{P}=0.0453$ ), with elevated levels present in older breast cancer patients, since age suppresses the functions of the immune system. The theory that age suppresses the functions of the immune system is defined as immune senescence (functional impairments of immunity) and is an important $\mathrm{T}$ cell defect associated with ageing, leading to higher susceptibility to infections or cancer in the elderly $(25,26)$.

In the present study, an association was observed between PD-1 levels and tumor size prior to surgery and intraoperatively $(\mathrm{P}=0.07$ and $\mathrm{P}=0.08$, respectively), with higher levels of $\mathrm{PD}-1$ present in T2 tumors compared with T1 tumors, which is consistent with the association of immune suppression with more advanced cancer; however, the differences were not statistically significant. These observations are concordant with those of Stagg et al (27), who concluded that blockade of PD-1 reduced the tumor size in a number of experimental cancer models. Similarly, Brahmer et al (28) reported good tumor responses to anti-PD-1 therapy in $37.5 \%$ of patients with melanoma, renal cell carcinoma, prostate cancer, non-small cell lung cancer and colorectal cancer, in a phase II clinical trial. Wolchok et al (29) demonstrated a strong response to a human antibody blocking PD-1 (nivolumab), administered concurrently with ipilimumab (anti-CTLA-4) in a substantial proportion of treated patients, with $\geq 80 \%$ tumor size regression. Topalian et al (30) identified that nivolumab therapy in melanoma patients produced durable responses.

An intriguing observation of the present study, in light of the paradigm shift in the extent of axillary dissection in breast cancer, was a decrease in PD-1 levels following the harvest of $\mathrm{SN}(\mathrm{s})$ containing metastases, in contrast to SN-negative patients $(\mathrm{P}=0.05)$. This implies that the removal of metastatic lymph nodes may affect immune surveillance, altering immune checkpoints. Furthermore, removal of healthy, non-malignant sentinel lymph nodes may potentially be harmful, as they appear to be significant in immune surveillance and essential for the elimination of cancer cells, particularly in early-stage breast cancer. Due to the small sample size of the present study, the results must be considered preliminary and should be interpreted with caution. However, they provide a basis for further investigation of the role of immune checkpoints in breast cancer, as well as potential justification on immunological grounds for purely surgical procedures, particularly with respect to axillary lymph node surgery. In the present study, all the patients with early-stage breast cancer were subjected to SNB, in accordance with the current standards of care (31). A report is expected in 2022 from the Sentinel node vs Observation after axillary UltraSouND (SOUND) trial $(6,7)$, confirming whether an abandonment of nodal procedures is a safe approach in selected patients. Therefore, no data comparing immune checkpoints in cohorts with completely intact axillary lymph nodes is available, at present; this is currently only feasible in patients who have positive or negative SNs. Gentilini et al (6), as part of the SOUND trial, investigated the outcome of breast cancer patients that did not undergo axillary surgery and preoperative ultrasound of the axilla, in order to identify patients with suspected lymph node involvement. The present study attempted to provide an immunological rationale for SN surgery on the basis of its histopathological status. In the SOUND trial, Gentilini et al are currently exploring the role of stem cells in the $\mathrm{SN}$ in breast cancer progression and recurrence. Vallacchi et al (32) demonstrated an increase in exhausted immune PD-1-positive cells in SNs of melanoma patients with more advanced disease. Overall, there is little data available to justify axillary lymph node surgery on immunological grounds and further investigation in larger studies is urgently required.

In the present study, a negative correlation between PD-1 expression and PR status following BCS and SNB ( $r=-0.39$; $\mathrm{P}=0.024)$ was identified. Cases with higher PR levels, which are associated with the luminal A subtype and good prognosis, exhibited lower PD-1 expression and, thus, decreased inhibition of the immune system.

The current study confirmed that breast cancer appears to be an immunogenic entity. In addition, renewed and increasing interest exists on the role of PR. The St. Gallen International Expert Consensus of 2013 (33) highlighted that PR added value in distinguishing between luminal $\mathrm{A}$ and luminal $\mathrm{B}$ subtypes, derived from the work of Prat et al (34) in which a cut-off point of $\geq 20 \%$ PR-positive tumor cells corresponded to luminal A tumors. During the San Antonio Breast Cancer Symposium (December 2013), Carroll et al (35) reported that $\mathrm{PR}$ is an essential prerequisite for the binding of ER with genes of good prognosis, whilst loss of PR appears to be associated with ER dysfunction. The findings of the present study that the highest levels of inhibitory regulators PD-1 occurred in PR-negative patients are consistent with those of Loi, Denkert, von Minckwitz et al (13), indicating that a targeted anti-PD-1 approach may be useful in triple negative and non-luminal HER2-positive breast cancer patients, such as patients without PR expression.

In conclusion, the present study demonstrated that breast cancer patients exhibited an altered profile of immune checkpoint markers, with higher concentrations of PD-1 in larger and PR-negative tumors. Surgical removal of lymph nodes containing tumor cells alters the immunologic profile by diminishing PD-1 levels. Breast cancer has not traditionally been considered immunogenic. However, increasing evidence indicates that the immunogenicity is important in certain molecular subtypes of breast cancer (36). The results of the present study provide a basis for further investigation of the role of immune checkpoints in breast cancer and immunological justification for purely surgical procedures, particularly axillary lymph node surgery. Finely-tuned modulation of the immune system may play a role in the treatment of breast cancer patients, enhancing the effects of the already well-established multimodality treatments (chemotherapy, endocrine therapy and anti-HER2 therapy), and may have an impact on axillary lymph node surgery. Therefore, further research in a large cohort of patients is required (37-40).

\section{Acknowledgements}

The authors would like to thank Professor Andrew Shorthouse, the Emeritus Professor of Surgery, University of Sheffield, for proofreading the manuscript. 


\section{References}

1. Urban JA and Baker HW: Radical mastectomy in continuity with en bloc resection of the internal mammary lymph-node chain; a new procedure for primary operable cancer of the breast. Cancer 5: 992-1008, 1952.

2. Halsted WS: I. The Results of Operations for the Cure of Cancer of the Breast Performed at the Johns Hopkins Hospital from June, 1889, to January, 1894. Ann Surg 20: 497-555, 1894.

3. Giuliano AE, Kirgan DM, Guenther JM and Morton DL: Lymphatic mapping and sentinel lymphadenectomy for breast cancer. Ann Surg 220: 391-398, 1994.

4. Giuliano AE, Hunt KK, Ballman KV, Beitsch PD, Whitworth PW, Blumencranz PW, Leitch AM, Saha S, McCall LM and Morrow M: Axillary dissection vs no axillary dissection in women with invasive breast cancer and sentinel node metastasis: a randomized clinical trial. JAMA 305: 569-575, 2011.

5. Galimberti V, Cole BF, Zurrida S, et al; International Breast Cancer Study Group Trial 23-01 investigators: Axillary dissection versus no axillary dissection in patients with sentinel-node micrometastases (IBCSG 23-01): a phase 3 randomised controlled trial. Lancet Oncol 14: 297-305, 2013.

6. Gentilini $\mathrm{O}$ and Veronesi U: Abandoning sentinel lymph node biopsy in early breast cancer? A new trial in progress at the European Institute of Oncology of Milan (SOUND: Sentinel node vs Observation after axillary UltraSouND). Breast 21: 678-681, 2012

7. Reimer T, Hartmann S, Stachs A and Gerber B: Local treatment of the axilla in early breast cancer: Concepts from the national surgical adjuvant breast and bowel project B-04 to the planned intergroup sentinel mamma trial. Breast Care Basel 9: 87-95, 2014

8. Pardoll DM: The blockade of immune checkpoints in cancer immunotherapy. Nat Rev Cancer 12: 252-264, 2012.

9. Topalian SL, Hodi FS, Brahmer JR, et al: Safety, activity, and immune correlates of anti-PD-1 antibody in cancer. N Engl J Med 366: 2443-2454, 2012.

10. Ott PA, Hodi FS and Robert C: CTLA-4 and PD-1/PD-L1 blockade: New immunotherapeutic modalities with durable clinical benefit in melanoma patients. Clin Cancer Res 19: 5300-5309, 2013

11. Eggermont AM, Spatz A and Robert C: Cutaneous melanoma. Lancet 383: 816-827, 2014.

12. Lipson EJ, Sharfman WH, Drake CG, et al: Durable cancer regression off-treatment and effective reinduction therapy with an anti-PD-1 antibody. Clin Cancer Res 19: 462-468, 2013.

13. Loi S: Tumor infiltrating lymphocytes (TILs) indicate trastuzumab benefit in early-stage HER2-positive breast cancer $\left(\right.$ HER $^{+}$BC). Presented at the San Antonio Breast Cancer Symposium, 2013.https://www.conferencenotes.co/conferences/ 5467ecb588d6868d254609f6/presentations/54739f542e0507a22 d1b9341?referrer=history.

14. Beavis PA, Milenkovski N, Henderson MA, et al: Adenosine receptor $2 \mathrm{~A}$ blockade increases the efficacy of anti-PD1 through enhanced antitumor T-cell responses. Cancer Immunol Res: Feb 11, 2015 (Epub ahead of print).

15. Denkert C, von Minckwitz G, Brase JC, et al: Tumor-infiltrating lymphocytes and response to neoadjuvant chemotherapy with or without carboplatin in human epidermal growth factor receptor 2-positive and triple-negative primary breast cancers. J Clin Oncol 33: 983-991, 2015.

16. Verbrugge I, Hagekyriakou J, Sharp LL, et al: Radiotherapy increases the permissiveness of established mammary tumors to rejection by immunomodulatory antibodies. Cancer Res 72 3163-3174, 2012.

17. Bos PD, Plitas G, Rudra D, Lee SY and Rudensky AY: Transient regulatory $\mathrm{T}$ cell ablation deters oncogene-driven breast cancer and enhances radiotherapy. J Exp Med 210: 2435-2466, 2013.

18. Vonderheide RH, LoRusso PM, Khalil M, et al: Tremelimumab in combination with exemestane in patients with advanced breast cancer and treatment-associated modulation of inducible costimulator expression on patient $\mathrm{T}$ cells. Clin Cancer Res 16: 3485-3494, 2010

19. Elston CW and Ellis IO: Pathological prognostic factors in breast cancer.I.The value of histological grade in breast cancer: Experience from a large study with long-term follow-up. Histopathology 19 403-410, 1991.

20. Allred DC, Harvey JM, Berardo M and Clark GM: Prognostic and predictive factors in breast cancer by immunohistochemical analysis. Mod Pathol 11: 155-168, 1998.
21. Wolff AC, Hammond ME, Hicks DG, Dowsett M, McShane LM, Allison KH, Allred DC, Bartlett JM, Bilous M, Fitzgibbons P, et al; American Society of Clinical Oncology; College of American Pathologists: Recommendations for human epidermal growth factor receptor 2 testing in breast cancer: American Society of Clinical Oncology/College of American Pathologists clinical practice guideline update. J Clin Oncol 31: 3997-4013, 2013.

22. Legat A, Speiser DE, Pircher H, Zehn D and Fuertes Marraco SA: Inhibitory receptor expression depends more dominantly on differentiation and activation than "exhaustion" of human CD8 T cells. Front Immunol 4: 455, 2013.

23. Poschke I, De Boniface J, Mao Y and Kiessling R: Tumor-induced changes in the phenotype of blood-derived and tumor-associated $\mathrm{T}$ cells of early stage breast cancer patients. Int J Cancer 131: $1611-1620,2012$

24. Azim HA, Brohée S, Peccatori FA, et al: Biology of breast cancer during pregnancy using genomic profiling. Endocr Relat Cancer 21: 545-554, 2014.

25. Poland GA, Ovsyannikova IG, Kennedy RB, Lambert ND and Kirkland JL: A systems biology approach to the effect of aging, immunosenescence and vaccine response. Curr Opin Immunol 29: 62-68, 2014.

26. Martinet KZ, Bloquet S and Bourgeois C: Ageing combines CD4 $\mathrm{T}$ cell lymphopenia in secondary lymphoid organs and $\mathrm{T}$ cell accumulation in gut associated lymphoid tissue. Immun Ageing 11: 8, 2014.

27. Stagg J, Andre F and Loi S: Immunomodulation via chemotherapy and targeted therapy: A new paradigm in breast cancer therapy? Breast Care Basel 7: 267-272, 2012.

28. Brahmer JR, Drake CG, Wollner I, et al: Phase I study of single-agent anti-programmed death-1 (MDX-1106) in refractory solid tumors: Safety, clinical activity, pharmacodynamics, and immunologic correlates. J Clin Oncol 28: 3167-3175, 2010.

29. Wolchok JD, Kluger H, Callahan MK, et al: Nivolumab plus ipilimumab in advanced melanoma. N Engl J Med 369: $122-133,2013$

30. Topalian SL, Sznol M, McDermott DF, et al: Survival, durable tumor remission, and long-term safety in patients with advanced melanoma receiving nivolumab. J Clin Oncol 32: 1020-1030, 2014

31. National Comprehensive Cancer Network: Clinical Practice Guidelines in Oncology. Version 2. 2015. http://www.nccn.org/ professionals/physician_gls/f_guidelines.asp\#breast. Accessed March 11, 2015.

32. Vallacchi V, Vergani E, Camisaschi C, et al: Transcriptional profiling of melanoma sentinel nodes identify patients with poor outcome and reveal an association of CD30(+) T lymphocytes with progression. Cancer Res 74: 130-140, 2014.

33. Goldhirsch A, Winer EP, Coates AS, et al; Panel members: Personalizing the treatment of women with early breast cancer: Highlights of the St Gallen International Expert Consensus on the Primary Therapy of Early Breast Cancer 2013. Ann Oncol 24: 2206-2223, 2013

34. Prat A, Cheang MC, Martín M, et al: Prognostic significance of progesterone receptor-positive tumor cells within immunohistochemically defined luminal A breast cancer. J Clin Oncol 31: 203-209, 2013.

35. Carroll JS: Steroids, nuclear receptors and breast cancer. Preface. Mol Cell Endocrinol 382: 623, 2014.

36. Denkert C: The immunogenicity of breast cancer - molecular subtypes matter. Ann Oncol 25: 1453-1455, 2014.

37. Loi S, Michiels S, Salgado R, et al: Tumor infiltrating lymphocytes are prognostic in triple negative breast cancer and predictive for trastuzumab benefit in early breast cancer: Results from the FinHER trial. Ann Oncol 25: 1544-1550, 2014

38. von Minckwitz G, Schneeweiss A, Loibl S, et al: Neoadjuvant carboplatin in patients with triple-negative and HER2-positive early breast cancer (GeparSixto; GBG 66): A randomised phase 2 trial. Lancet Oncol 15: 747-756, 2014.

39. Denkert C: Diagnostic and therapeutic implications of tumor-infiltrating lymphocytes in breast cancer. J Clin Oncol 31: 836-837, 2013.

40. Loi S, Sirtaine N, Piette F, et al: Prognostic and predictive value of tumor-infiltrating lymphocytes in a phase III randomized adjuvant breast cancer trial in node-positive breast cancer comparing the addition of docetaxel to doxorubicin with doxorubicin-based chemotherapy: BIG 02-98. J Clin Oncol 31: 860-867, 2013 